\title{
FRECUENCIA Y SEVERIDAD DE LA SEQUÍA EN LA PENÍNSULA DE YUCATÁN COMO INSTRUMENTO PARA EL ORDENAMIENTO DEL TERRITORIO
}

\author{
Jesús David GÓMEZ DÍAZ ${ }^{1}$, Alejandro Ismael MONTERROSO RIVAS ${ }^{1}$, \\ Lizeth Margarita LECHUGA GAYOSSO ${ }^{1}$ \\ ${ }^{1}$ Departamento de Suelos, Universidad Autónoma Chapingo. México. \\ dgomez1059@yahoo.com.mx, aimrivas@correo.chapingo.mx
}

\section{RESUMEN}

Se presenta un estudio del comportamiento de la sequía en cinco estaciones meteorológicas de la Península de Yucatán, México (norte, centro-oeste, centro-este, oeste y este) para el periodo de 1961 al 2012 (52 años). Se evaluó a partir de datos de precipitación media anual aplicando el criterio del Standar Precipitation Index (SPI), identificándose la frecuencia e intensidad de la sequía en el periodo de estudio. Adicionalmente se cotejó la severidad de la sequía para cada estación con la declaratoria de sequía por municipios entre el 2000 al 2012 reportadas por CENAPRED. Para el periodo de estudio, la estación ubicada al oeste presento algún grado de sequía en 15 años (4 ligeras, 6 moderadas, 2 muy secos, 2 extremas y 1 excepcional), la estación de la región norte presentó algún grado de sequía también en 15 años (4 ligeras, 6 moderadas, 2 muy secas, 2 extremas y 1 excepcional). Las estaciones de la región centro también presentaron 15 años con sequía, la centro-oeste presentó 5 ligeras, 5 moderadas, 2 muy secas, 2 extremas, 1 excepcional, mientras que la región centro-este presento 4 ligeras, 6 moderadas, 2 muy secas, 2 extremas y 1 excepcional. La estación de la región este presentó 16 años con sequía (5 ligeras, 6 moderadas, 2 muy secas, 2 extremas, 1 excepcional). Aunque el número de años con sequia es similar, la mayoría de las veces no coincide la severidad de la sequía para un mismo año en las regiones de la Península de Yucatán.

Palabras clave: sequía, península de Yucatán, Standar Precipitation Index, CENAPRED.

\begin{abstract}
A study of the behavior of drought is presented in five weather stations of the Yucatan Peninsula, Mexico (northern, centra-west, central-east, west and east) for the period 1961 to 2012 (52 years). It was evaluated data from annual rainfall using the criteria of Standard Precipitation Index (SPI), identifying the frequency and intensity of drought in the study period. In addition, the severity of the drought for every season with the declaration of drought by municipalities from 2000 to 2012 reported by CENAPRED was checked. For the study period, the station located in the west region, presented 15-year drought (4 light, 6 moderate, 2 very dry, 2 extreme, 1 exceptional), the station in the north region had some degree of drought also in 15 years (4 light, 6 moderate, 2 very dry, 2 extreme and 1 exceptional). The meteorological stations of the central region also had 15-year with some degree of drought, the central-west had 5
\end{abstract}


light, 5 moderate, 2 very dry, e exteme and 1 exceptional, while the central east had 4 lights, 6 moderate, 2 very dry, 2 extreme, and 1 exceptional). The station located at the east region had 16 years with some degree of drought ( 5 light, 6 moderate, 2 very dry, 2 extreme, and 1 exceptional). Although the number of years with drought is similar in all the stations, most of the time does not match the severity of the drought for the same year in the regions of the Yucatan Peninsula.

Key words: drought, Yucatan Peninsula, Standard Precipitation Index, CENAPRED.

\section{INTRODUCCIÓN}

La sequía es un fenómeno natural que tiene impactos adversos en los humanos y en los ecosistemas (Rhee y Carbone, 2011). Es una característica del clima y es diferente a la aridez (Wilhite y Buchanan-Smith, 2005). La sequía es un fenómeno natural que puede afectar virtualmente todos los regímenes climáticos. Generalmente se define a la sequía como una deficiencia en la precipitación relativa con respecto a la que se espera (normal), que cuando se extiende en una estación o un periodo más largo de tiempo resulta en la incapacidad de satisfacer las demandas de agua en las actividades humanas y en el medio ambiente (Hayes et al., 2011). La estimación de los índices de sequía se hace a partir de las condiciones normales y requieren de datos históricos con un periodo mayor a 30 años para examinar los promedios climatológicos en un plazo largo (Rhee y Carbone, 2011). Los índices de sequía más comúnmente usados en diferentes partes del mundo son el Índice de Severidad de la Sequía de Palmer (ISSP) y el Índice Estandarizado de Precipitación (SPI) (Rhee y Carbone, 2011). El ISSP desarrollado por Palmer (1965) indica la severidad física de la sequía por la falta de humedad en el suelo y es considerado generalmente útil en la agricultura y otros usos del agua que son sensibles a la falta de humedad almacenada en el suelo (Guttman, 1998). Sin embargo requiere de varios parámetros para ser estimada, varios de los cuales no se reportan en países con deficiencias en el sistema de monitoreo de la intensidad de la lluvia y de parámetros de humedad del suelo, como es el caso de México, aunado a esto, varios estudios de referencia sobre sus propiedades y sensibilidad han mostrado que la asunción de estandarización en realidad no es válida ya que las comparaciones espaciales y temporales tienen desviaciones que conducen a conclusiones erróneas (Alley, 1984; Heddinghaus y Sabol, 1991; Karl, 1983, 1986; Guttman, 1991, y Guttman et al., 1992).

Por otra parte, el SPI lo desarrolló McKee et al., (1993) y estima el déficit de precipitación a diferentes tiempos y escalas y fue propuesto con el objetivo de dar una mejor representación a la sequía y a los periodos húmedos comparado con el Índice de Palmer (Guttman 1998, 1999). El SPI es un índice de probabilidad que considera únicamente a la precipitación, en contraste con otros que definen la sequía y que requieren variables como temperatura o evapotranspiración. Se basa en la probabilidad de ocurrencia de una cantidad de lluvia donde un valor de cero indica la mediana de precipitación recibida, es decir, la mitad de la precipitación histórica se encuentra por arriba y la otra mitad se encuentra por debajo. El índice es negativo en la definición de la sequía y positivo para condiciones de humedad. Mientras los valores de lluvia registrada se hacen más extremos así el índice se hace más positivo o negativo. El SPI 
se puede calcular para diversas escalas, hasta para 48 meses continuos o incluso para un solo mes, capturando así diferentes escalas espacio-temporales que definen los tipos de sequía (Edwards y McKee, 1997), los intervalos de tiempo cortos del orden de meses pueden ser importantes para la agricultura, mientras los intervalos largos que comprenden años pueden ser importantes para el manejo de las reservas de agua a nivel local y regional (Guttman, 1998).

Este índice es apropiado para monitorear la sequía en grandes áreas dado que sus valores son comparables en el espacio y en el tiempo como consecuencia del uso del proceso de transformación estandarizada de la precipitación en su estimación (Guttman 1998, 1999). En este contexto, la OMM y la CNULD recomiendan el uso amplio del Índice de Precipitación Estandarizada (SPI) en los países que deseen monitorear la sequía y que éste sea el índice estándar disponible a nivel mundial en adición a los índices e indicadores locales (Hayes et al., 2011). En este contexto se seleccionó el SPI para analizar la frecuencia y severidad de la sequía a nivel anual para la Península de Yucatán, a partir de los datos de precipitación media anual de cuatro estaciones representativas de las regiones norte, sur, este y oeste de la zona de estudio.

\section{MÉTODOS}

Para realizar este análisis, se obtuvieron los datos de precipitación de cinco estaciones del Servicio Meteorológico Nacional (SMN) de enero de 1960 a diciembre de 2012. La información mostró datos faltantes y observaciones incongruentes, por lo que fue necesario estimar los datos faltantes y recalcular los datos con valores incongruentes con el procedimiento modificado de Normal-Ratio Method definido por Paulhus y Kohler (1952). Para la determinación del SPI se utilizó el procedimiento establecido por McKee et al., (1993) y para su clasificación, se utilizó el criterio establecido por CONAGUA-SMN (2013) tal y como se muestra en la figura 1:

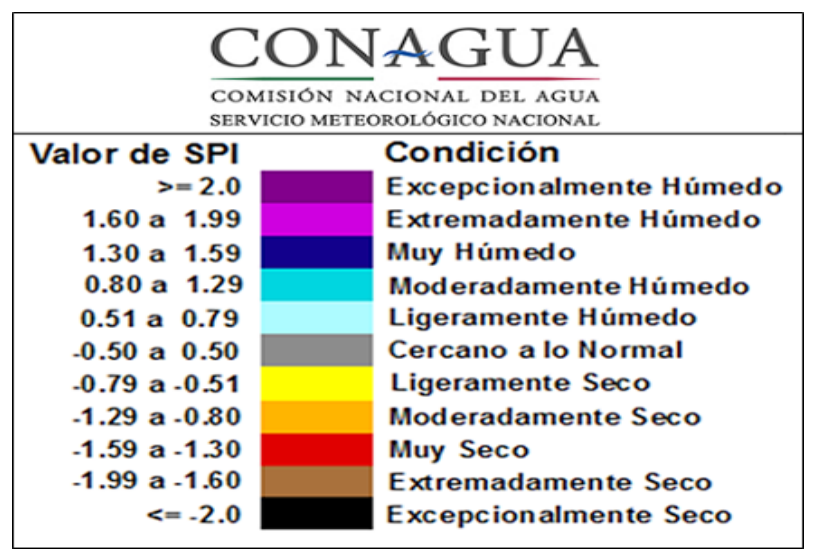

Fig. 1: Clasificación del Índice de Precipitación Estandarizada (SPI) por CONAGUA-SMN (2013)

Adicionalmente se cotejo la severidad de la sequía para cada estación con la declaratoria de sequía por municipios del 2000 al 2012 reportadas por CENAPRED (2012). 


\section{RESULTADOS}

En la tabla 1 se muestran los datos generales de ubicación y de precipitación media anual y las mensuales de las cinco estaciones de referencia para este estudio. La estación representativa de la zona oeste es Campeche, Camp. (4038), la de la zona norte es Río Lagartos, Yuc. (31024), la de la zona centro-oeste es Muna, Yuc. (31021), de la zona centro-este es Oxkutxcab, Yuc.( 31061) y de la zona este es la estación de Felipe Carrillo Puerto, Q.R. (23003).

En la figura 2 se muestra la frecuencia de condiciones de humedad y sequía estimada para la precipitación anual para las estaciones de referencia agrupadas por décadas desde 1961 al 2010 y para los años 2011 y 2012.

En el cuadro 2 se presenta un concentrado de las sequías por grado de intensidad que se presentaron en las estaciones de las diferentes regiones de la Península de Yucatán.

De los 42 años que comprendió la estimación de la ocurrencia de sequías, los años caracterizados con sequías para las estaciones de las diferentes regiones fue como sigue:

Región Oeste, 15 años presentaron sequías de los cuales 4 fueron ligeras, 6 moderadas, 2 muy secas, 2 extremas y 1 excepcional

Región Norte, 15 años fueron caracterizados con sequía, con 4 años como ligera, 6 como moderada, 2 muy secos, 2 extrema y 1 excepcionalmente seco

Región Centro-Oeste, 15 años caracterizados con sequía, de los cuales 5 como ligera, 5 como moderada, 2 muy secos, 2 extremadamente secos y 1 excepcionalmente seco.

Región Centro-Este, 15 años presentaron sequía, de los cuales 4 como ligera, 6 como moderada, 2 muy secos, 2 extrema y 1 excepcionalmente seco.

Región Este, 16 años con sequía, de los cuales 5 como ligera, 6 moderada, 2 muy secos, 2 extremos, 1 excepcionalmente seco.

La declaratoria de contingencia por sequía del 2000 al 2011 para la Península de Yucatán fue solo para cuatro años de este periodo, con 5 municipios para el 2000, 3 municipios para el 2004, 49 municipios para el 2008 y 110 municipios para el 2009 (CENAPRED, 2012), cuando en las estimaciones, para el año 2000, se presentó sequia ligera en la estación de la región oeste y moderada para la estación de la región norte, en 2004 las estaciones que presentaron sequia fue la del oeste como ligera y la moderada para la centro-este, para el 2008 solo se estimó sequia ligera para la estación de la región este y para el 2009 la sequía fue más generalizada en la Península de Yucatán con condición de extrema para la estación este, moderada para las estaciones de las regiones oeste y la centro-este y ligera para la estación de la región centro-oeste. 


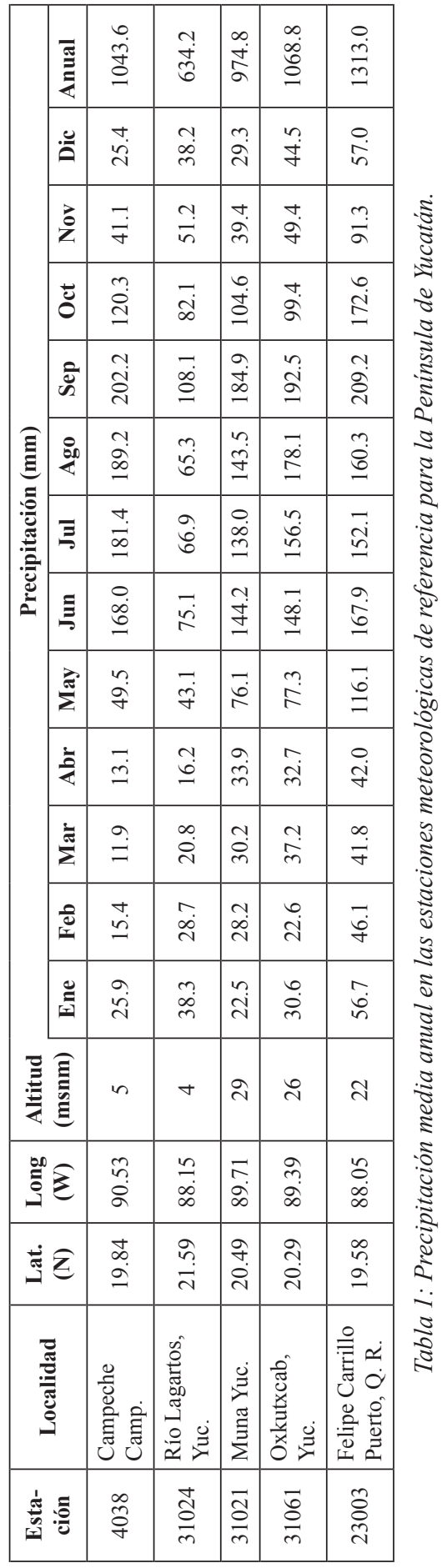

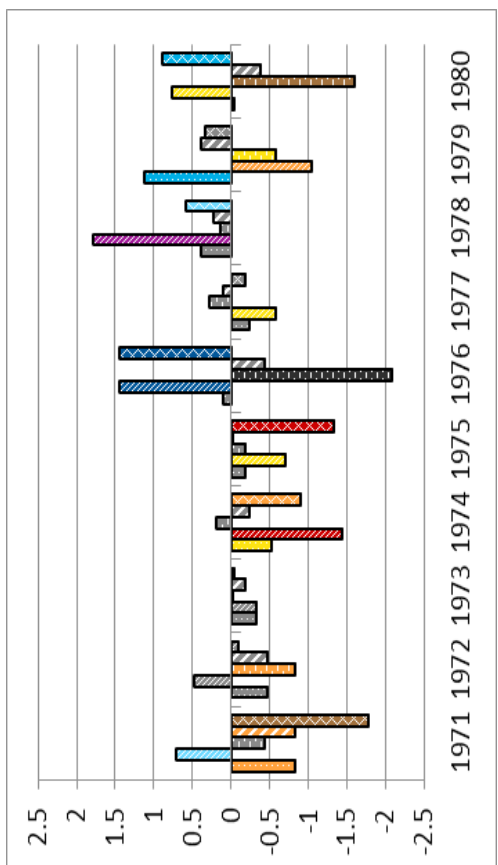



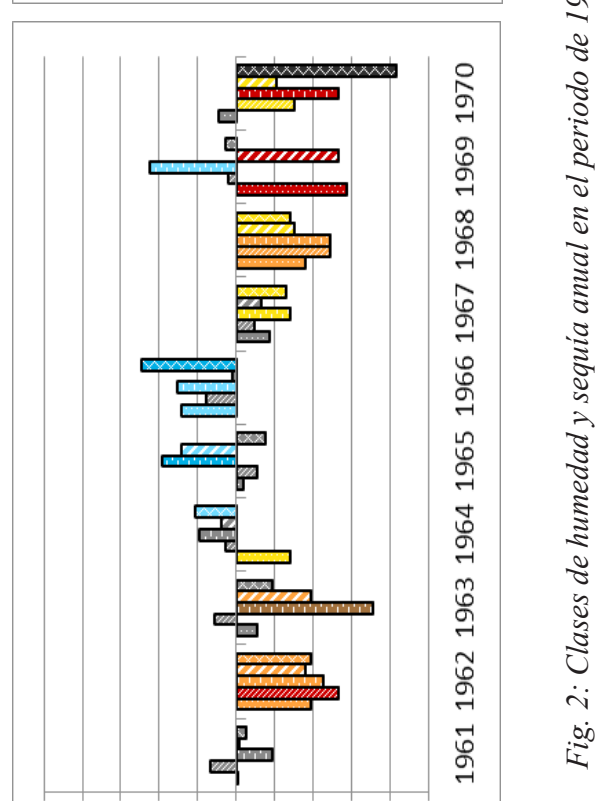

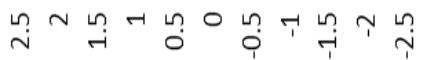



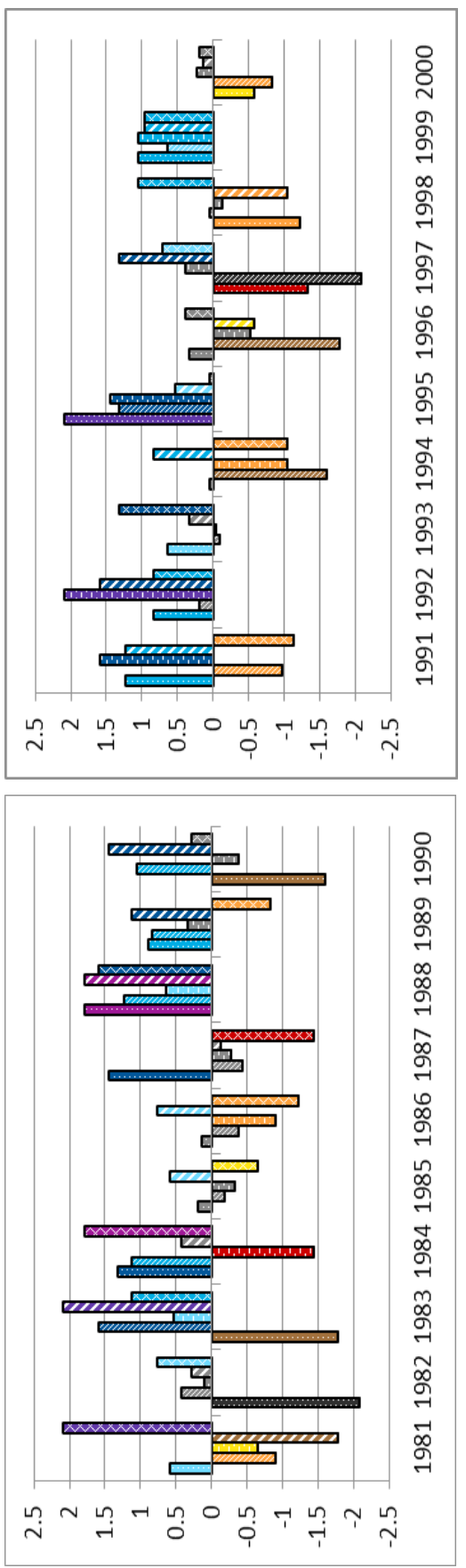
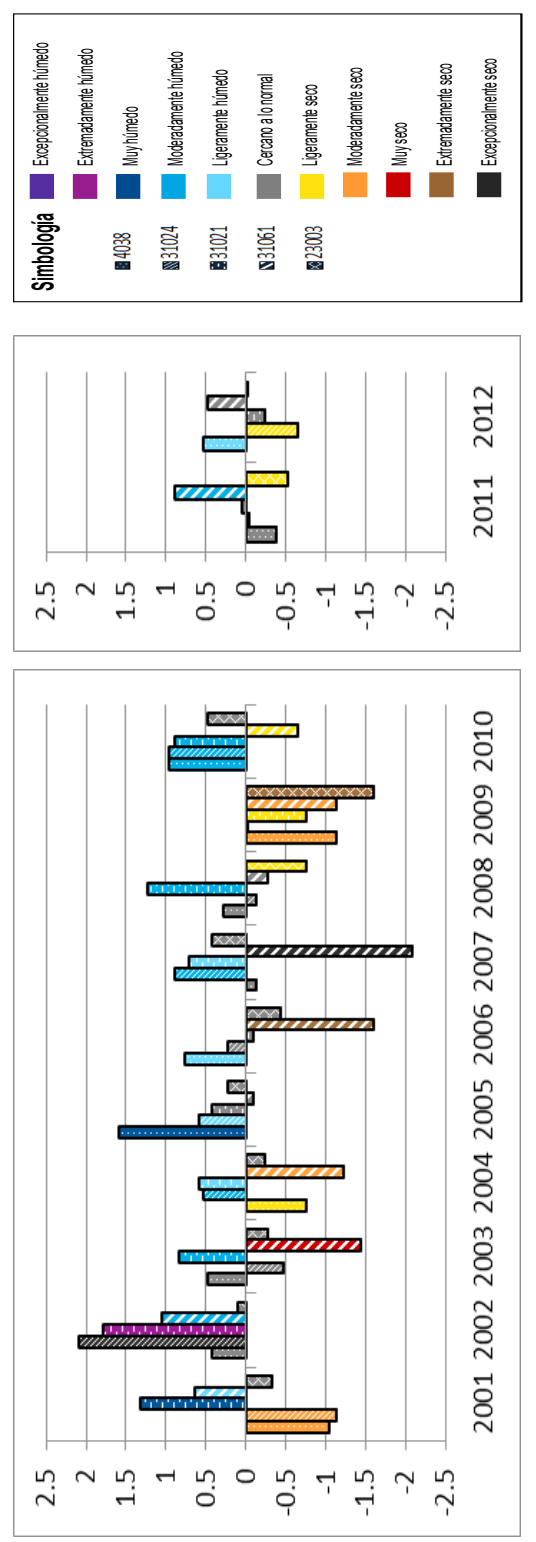

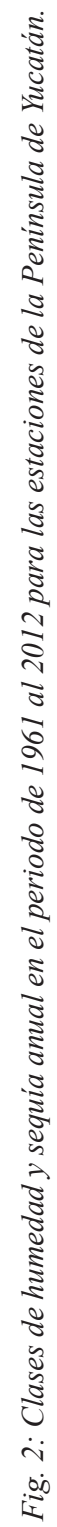




\begin{tabular}{|c|c|c|c|c|c|c|}
\hline \multirow{2}{*}{ Década } & \multirow{2}{*}{ Año } & \multicolumn{5}{|c|}{ Estación por región } \\
\hline & & Oeste & Norte & Centro-Oeste & Centro-Este & Este \\
\hline \multirow{7}{*}{$\begin{array}{l}1961- \\
1970\end{array}$} & 1962 & Mod. & Muy S. & Mod. & Mod. & Mod. \\
\hline & 1963 & - & - & Extrem. & Mod. & - \\
\hline & 1964 & Lig. & - & - & - & - \\
\hline & 1967 & - & - & Lig. & - & Lig \\
\hline & 1968 & Mod. & Mod. & Mod. & Lig. & Lig. \\
\hline & 1969 & Muy S. & - & - & Muy S. & - \\
\hline & 1970 & - & Lig. & Muy S. & Lig. & Exep. \\
\hline \multirow{8}{*}{$\begin{array}{l}1971- \\
1980\end{array}$} & 1971 & Mod. & - & - & Mod. & Extrem. \\
\hline & 1972 & - & - & Mod. & - & - \\
\hline & 1974 & Lig. & Muy S. & - & - & Mod. \\
\hline & 1975 & - & - & Excep. & - & - \\
\hline & 1976 & - & - & Excep. & - & - \\
\hline & 1977 & - & Lig. & - & - & - \\
\hline & 1979 & - & Mod. & Lig. & - & - \\
\hline & 1980 & - & - & Extrem. & - & - \\
\hline \multirow{9}{*}{$\begin{array}{l}1981- \\
1990\end{array}$} & 1981 & - & Mod. & Lig. & Extrem. & - \\
\hline & 1982 & Excep. & - & - & - & - \\
\hline & 1983 & Extrem. & - & - & - & - \\
\hline & 1984 & - & - & Muy S. & - & - \\
\hline & 1985 & - & - & - & - & Lig. \\
\hline & 1986 & - & - & Mod. & - & Mod. \\
\hline & 1987 & - & - & - & - & Muy S. \\
\hline & 1989 & - & - & - & - & Mod. \\
\hline & 1990 & Extrem. & - & - & - & - \\
\hline \multirow{6}{*}{$\begin{array}{l}1991- \\
2000\end{array}$} & 1991 & - & Mod. & - & - & Mod. \\
\hline & 1994 & - & Extrem & Mod. & - & Mod. \\
\hline & 1996 & - & Extrem & - & Lig. & - \\
\hline & 1997 & Muy S. & Excep. & Lig. & - & - \\
\hline & 1998 & Mod. & - & - & Mod. & - \\
\hline & 2000 & Lig. & Mod. & - & - & - \\
\hline \multirow{8}{*}{$\begin{array}{l}2001- \\
2010\end{array}$} & 2001 & Mod. & Mod. & - & - & - \\
\hline & 2003 & - & - & - & Muy S. & - \\
\hline & 2004 & Lig. & - & - & Mod. & - \\
\hline & 2006 & - & - & - & Extrem. & - \\
\hline & 2007 & - & - & - & Excep. & - \\
\hline & 2008 & - & - & - & - & Lig. \\
\hline & 2009 & Mod. & - & Lig. & Mod. & Extrem. \\
\hline & 2010 & - & - & - & Lig. & - \\
\hline \multirow{2}{*}{$\begin{array}{l}2011- \\
2012\end{array}$} & 2011 & - & - & - & - & Lig. \\
\hline & 2012 & - & Lig. & - & - & - \\
\hline
\end{tabular}

Tabla 1: Severidad de sequía en las estaciones meteorológicas de referencia por región para la Península de Yucatán. 


\section{DISCUSIÓN}

La Península de Yucatán es una plataforma de roca caliza relativamente plana, aunque con la interacción de varios sistemas atmosféricos con los que se asocian las precipitaciones en la región. De septiembre a mayo se tienen lluvias por el efecto de los frentes fríos que entran a la Península de Yucatán provenientes del norte, los que recogen humedad en el Golfo de México y generan el levantamiento de las masas de aire más cálido y con humedad. En la mitad caliente del año se presentan varios sistemas, los vientos provenientes del centro de alta presión del Golfo de México que tienen trayectorias del noreste al suroeste y que por la cercanía de este centro de alta presión, el norte de la Península tiene las precipitaciones menores, sin embargo cuando estos sistemas entran a tierra, al aumentar la convección, aumenta la precipitación como se aprecia en las estaciones de las regiones centro-oeste y centro-este, así como la del oeste. La región con mayor precipitación es la este asociada a la entrada de ondas tropicales, las cuales al ir avanzando sobre la Península de Yucatán, son empujadas por el sistema de vientos del centro de alta presión del Golfo de México y disminuye la precipitación por este fenómeno. En la temporada de Huracanes del Atlántico (1 de junio al 30 de noviembre), las tormentas tropicales y los Huracanes que llegan a pasar por la zona de influencia de la Península de Yucatán, generan precipitaciones abundantes, principalmente durante los meses de septiembre y octubre (Gómez et al., 2013). Por lo antes descrito es que se puede explicar la heterogeneidad en la ocurrencia de sequías en esta parte de México, que aunque fue similar el número de sequias (de 15 a 16) para las diferentes estaciones de las regiones descritas, solo en algunos años fue generalizada la sequía pero en la mayoría de los años con sequía solo en algunas regiones se caracterizaron como tal con diferentes intensidades.

\section{AGRADECIMIENTOS}

Al Centro de Investigación en Recursos Naturales y Medio Ambiente de la Universidad Autónoma Chapingo por el apoyo financiero para la realización del presente estudio

\section{REFERENCIAS}

Alley, W. M. 1984. The Palmer Drought Severity Index: Limitations and Assumptions, J. Clim. Appl. Meteor. 23: 1100-1109.

CENAPRED, 2012, Base de datos de sequías oficiales por municipios.

CONAGUA-SMN, 2013. Escala de medición de la sequía del SPI del monitor de sequía de América del Norte, Febrero del 2013.

Edwards, D. C. \& McKee, T. B., 1997, Characteristics of $20^{\text {th }}$ Century drought in the United States al multiple time scales, Climatology Rep. 97-2, Department of Atmospheric Science, Colorado State University, Fort Collins, Colorado.

Gómez, J.D., A.I. Monterroso, L.M. Lechuga. 2013. Comportamiento de la sequía a nivel regional en México en: CONAFOR-UACh. 2013. Línea base nacional de degradación de tierras y desertificación. Informe final. Comisión Nacional Forestal y Universidad Autónoma Chapingo. Zapopan, Jalisco. 
Guttman, N. B., 1991, Sensitivity of the Palmer Hydrologic Drought Index, Water Resources, Bull. 27:797-807.

Guttman, N. B., 1998, Comparing the Palmer Drought Index and the Standardized Precipitation Index. JAWRA Journal of the American Water Resources Association, 34: 113-121.

Guttman, N. B., 1999, Accepting the Standardized Precipitation Index: A Calculation Algorithm. JAWRA Journal of the American Water Resources Association, 35: 311-322.

Guttman, N. B., J. R. Wallis J. and R. M. Hosking, 1992, Spatial Comparability of the Palmer Drought Severity Index, Water Resources Bulletin 28: 1111-1119.

Hayes, M., Svoboda, M., Wall, N., \& Widhalm, M., 2001, The Lincoln declaration on drought indices: Universal meteorological drought index recommended. Bulletin of the American Meteorological Society, 92, 485-488.

Heddinghaus, T. B. and P. Sabol, 1991. A Review of the Palmer Drought Severity Index and Where Do We Go From Here? Proc. 7th Conf. on Applied Climatology, September 10-13, 1991, American Meteorological Society, Boston, Massachusetts, pp. 242-246

Karl, T. B., 1983. Some Spatial Characteristics of Drought Duration in the United States. J. Clim. Appl. Meteor. 22: 1356-1366.

Karl, T. R., 1986. The Sensitivity of the Palmer Drought Severity Index and Palmer's Z.Index to Their Calibration Coefficients Including Potential Evapotranspiration. J. Clim. Appi. Meteor. 25:77-86.

McKee, T. B., Doesken, N. J. \& Kleist, J., 1993. The relationship of drought frequency and duration to time scales, Anaheim: American Meteorological Society.

Palmer, W. C., 1965, Meteorological Drought. Research paper no.45, U.S. Department of Commerce Weather Bureau (58 pgs). Available online by the NOAA National Climatic Data Center at http://www.ncdc.noaa.gov/temp-and-precip/drought/ docs/palmer.pdf

Paulhus, H. A. \& Kohler, M. A., 1952. Interpolation of missing precipitation records. Monthly Weather Review, pp. 129-133.

Rhee, J. \& Carbone, G. J.,Estimating Drought Conditions for Regions with Limited Precipitation Data, Journal of Applied Meteorology and Climatology. Volume 50, Issue 3 (March 2011) pp. 548-559.

Wilhite, D. A. \& Buchanan-Smith, M. 2005, "Drought as Hazard: Understanding the Natural and Social Context" in Drought and Water Crises Science, Technology, and Management Issues, Editor D. A. Wilhite; Ed. Taylor \& Francis Group, Boca Raton, Florida, USA; 3-32, 406 pp. 